\title{
DEPTH PERCEPTION IN DRIVING: ALCOHOL INTOXICATION, EYE MOVEMENT CHANGES, AND THE DISRUPTION OF MOTION PARALLAX
}

\author{
Mark Nawrot \\ Department of Psychology \\ North Dakota State University \\ Fargo, North Dakota, USA \\ email: mark_nawrot@ndsu.nodak.edu
}

\begin{abstract}
Summary: Motion parallax, the ability to recover depth from retinal motion, is a crucial part of the visual information needed for driving. Recent work indicates that the perception of depth from motion parallax relies on the slow eye movement system. It is well known that that alcohol intoxication reduces the gain of this slow eye movement system, the basis for the "horizontal gaze nystagmus" field sobriety test. The current study shows that alcohol intoxication also impairs the perception of depth from motion parallax due to its influence on the slow eye movement system. Observer thresholds in both active and passive motion parallax tasks are significantly increased by acute alcohol intoxication. Perhaps such a failure of motion parallax plays a role when intoxicated drivers must make quick judgements with what could be inaccurate or missing perceptual information about the location of obstacles around them.
\end{abstract}

\section{INTRODUCTION}

Visual processing is of undeniable importance to driving. Drivers must be able to see objects and then extract information about the object, such as its meaning and its position and movement. To do this, drivers must constantly move their eyes so that the fovea, the area with the best visual acuity, is positioned upon the area of interest. These eye movements are produced by two different systems. The fast eye movement system generates saccadic or ballistic eye movements to foveate an item of interest. The slow eye movement system produces tracking eye movements to maintain fixation on an object during movement of the object or movement of the observer.

Alcohol's effect on both the fast and slow eye movement systems has been well studied (see Stapleton, Guthrie, \& Linnoila, 1986, for a review with considerations of traffic safety). Alcohol intoxication slows the initiation and the velocity of saccadic eye movements and reduces the gain of slow eye movements (Moser, Heide \& Kompf, 1998; Holdstock \& de Wit, 1999). Gain is a ratio of eye velocity / target velocity and should be close to 1.0 to maintain fixation on the moving target. With alcohol intoxication, the slow eye movements are too slow, gain is $<1.0$ and the visual system must recruit the fast eye movement to generate a "catch-up saccade." This produces the jerky eye movements, also called horizontal gaze nystagmus, that are an important component of field sobriety tests (Forkiotis, 1986, Tiffany, 1986; Good \& Augsburger, 1986; Belton 1987).

The specific effects of alcohol intoxication on visual perceptual performance are also well known. For instance, contrast sensitivity is affected by alcohol (Nicholson et al., 1995; Pearson \& Timney, 1998) but visual acuity and color vision remain largely unaffected by alcohol (Wallgren \& Barry, 1970; Watten \& Lie, 1996, Hill and Tofollon, 1990). There remains controversy regarding alcohol's effect on depth perception from binocular stereopsis (Hill \& Toffolon, 1990; Wratten \& Lie, 1996). 
Wegner and Fahle (1999) suggest the discrepancy is due to large interindividual differences and floor effects in previous binocular stereopsis studies hiding the small changes caused by alcohol intoxication.

Although binocular stereopsis has received a lot of study, the perception of depth from motion parallax is a much more important in situations such as driving. Motion parallax is created when an observer translates through the environment. During this translation, the visual system maintains fixation on a particular object by making a slow eye movement in the direction opposite the translation. From the the observer's view, objects nearer than fixation, although remaining stationary in the environment, change in relative position in the direction opposite the translation. Conversely, objects farther away than fixation change relative position in the direction of observer translation. This motion parallax, or changes in relative position of objects at different distances, also creates movement on the observer's retina. The object of fixation remains stationary on the retina, nearer objects move one way, and distant objects move the opposite direction. For the perception of depth from motion parallax, the visual system relies on these retinal motions. However, since these retinal motion are ambiguous the visual system also relies on slow eye movement system to provides "extra-retinal" information to distinguish which direction of retinal motion corresponds to near or far depth (Nawrot, 1997). It also appears that perceived depth is scaled with the gain or velocity of these eye movements (Nawrot, 2000). While it is well known that alcohol intoxication reduces the gain of slow eye movements, it is unknown whether alcohol intoxication impairs the perception of depth from motion parallax due to its influence on the eye movement system.

\section{METHOD}

A repeated measures design was used to assess the effect of alcohol intoxication on eye movements and the perception of depth from motion parallax. Basic visual function screening included visual acuity, contrast sensitivity (Pelli-Robson chart), and stereopsis screening tests including Randot and Stereo Fly tests. Eye movements were measured with a head mounted Skalar infra-red Limbus eye tracker recording from the right eye. The left eye was patched. Eye movements were measured when observers viewed a small dot traversing the computer monitor with a sinusoidal velocity pattern of 11, 22, and $33 \mathrm{deg} / \mathrm{sec}$. Depth perception thresholds were determined for binocular stereopsis, active and passive motion parallax. The stimuli for all tests were computer generated random-dot patterns appearing as a corrugated surface if the depth information in the stimulus was perceived by the observer (Rogers \& Graham, 1979). Binocular stereoscopic presentation of the stimulus was accomplished through frame sequential presentation using ferro-electric shutters mounted in trial frames worn by the observers. For active motion parallax, observers moved their head along the interaural axis with their chin in a rest that moving along a linear head tracking device. Every few milliseconds head position was measured to the nearest $0.1 \mathrm{~mm}$ and changes to the random-dot stimulus were made: dots to appear nearer were shifted in the direction opposite the head movement, dots to appear farther were shifted in the direction of the head movement, and dots at fixation remained unchanged. With this stimulus design, dots drawn on the flat screen of the monitor appeared as a corrugated surface to a normal observer (Figure 1). For passive motion parallax presentations, observers were seated and moved by the experimenter along a linear track system which gave observer position to the nearest $0.5 \mathrm{~mm}$. In all tests, observers performed a $2 \mathrm{AFC}$ task, making a decision regarding the depth perceived in the random-dot stimulus. The amount of depth in the stimulus was varied in successive trials using a staircase procedure to determine each observer's threshold, the smallest amount of depth for which they could still reliably see depth. In the 
intoxicated condition, observers were administered ethyl alcohol at $0.8 \mathrm{~g}$ per $\mathrm{kg}$ body weight, as a mixture of 100 proof Vodka and orange juice, to achieve a blood alcohol content (BAC) near $0.1 \%$.

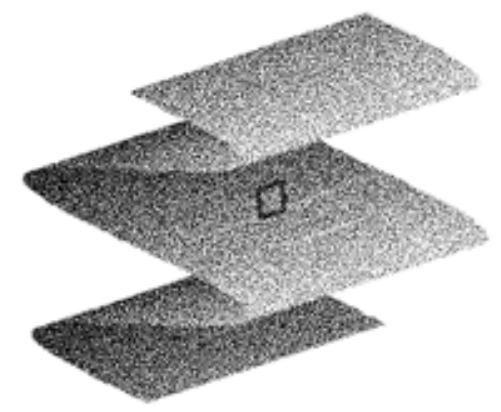

Figure 1. The random-dot stimulus, when perceived in depth due to binocular stereopsis or motion parallax, appeared as a corrugated surface coming out of the screen or receding back into the screen. The phase of the corrugation varied between trials in relation to the fixation mark. The observer's task was to report which portion of the stimulus appeared to recede away in depth. In the illustration above the correct answer is the portion just above the fixation mark.

\section{RESULTS}

As expected, alcohol intoxication reduced eye movement gain. First, eye movement recordings were analyzed to determine the effect of alcohol intoxication on the slow eye movement system. Figure 2 shows a representative eye position recording from a sober observer and an intoxicated observer.
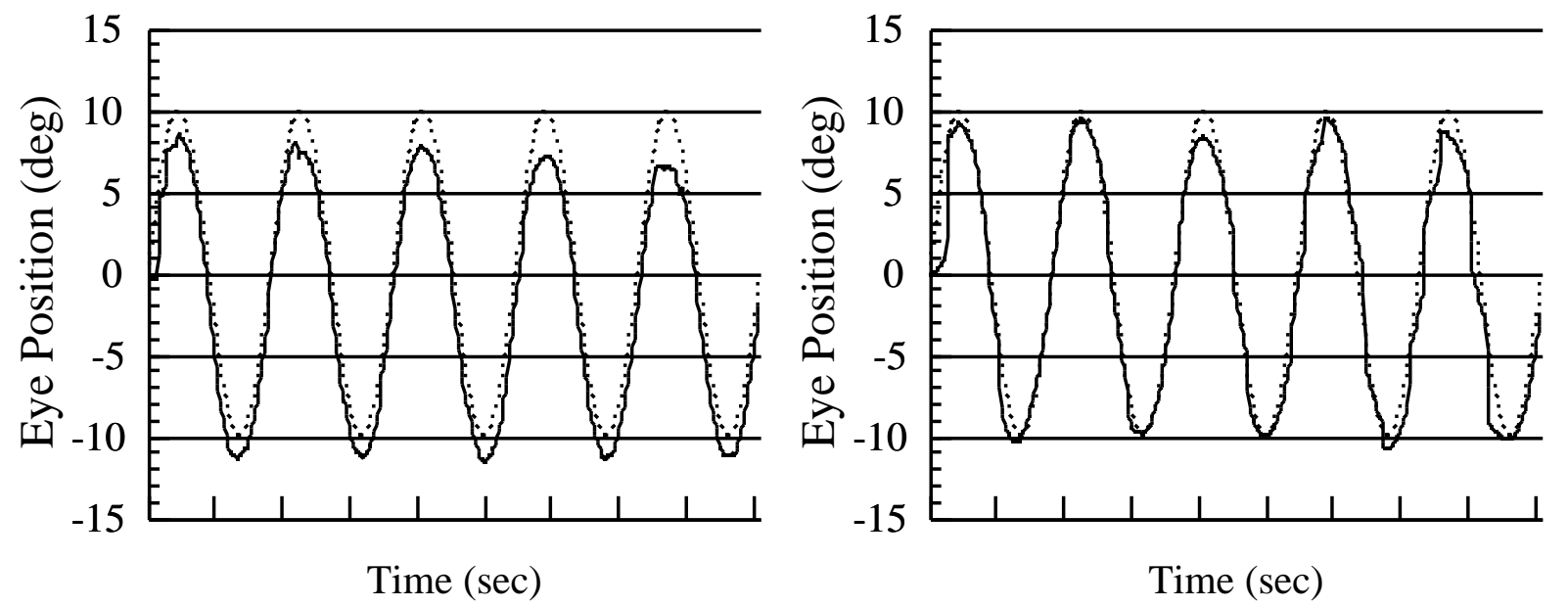

Figure 2. A representative eye position recording for the $22 \mathrm{deg} / \mathrm{sec}$ sinusoid target for a sober (left) and intoxicated observer (right). Target position is shown with the dotted line, eye position with the solid line. The sober observer's head moved between calibration and recording causing an overall shift in the function, but the pursuit is still much more accurate than that shown by the intoxicated observer.

For each eye position recording (e.g., Figure 2), instantaneous velocity was calculated for each recording interval $(15 \mathrm{msec})$ and compared to the sinusoidal target velocity at that interval. A ratio of the two velocities (e.g., eye/target) is called gain and should be very close to 1.0 for accurate pursuit. Gain values were calculated for the central 17 degrees of eye position, excluding the last 1.5 degrees 
when both eye and target were slowing, reversing, and then accelerating. Figure 3 shows the gain for the two eye position recording shown previously. Sober average gain values are between 0.95 and 0.98 while intoxicated gain values are.between 0.6 and 0.7 . Intoxicated observer also show numerous "catch-up" saccades where the fast eye movement system is recruited to jerk the eyes into to a position to resume pursuit of the target.
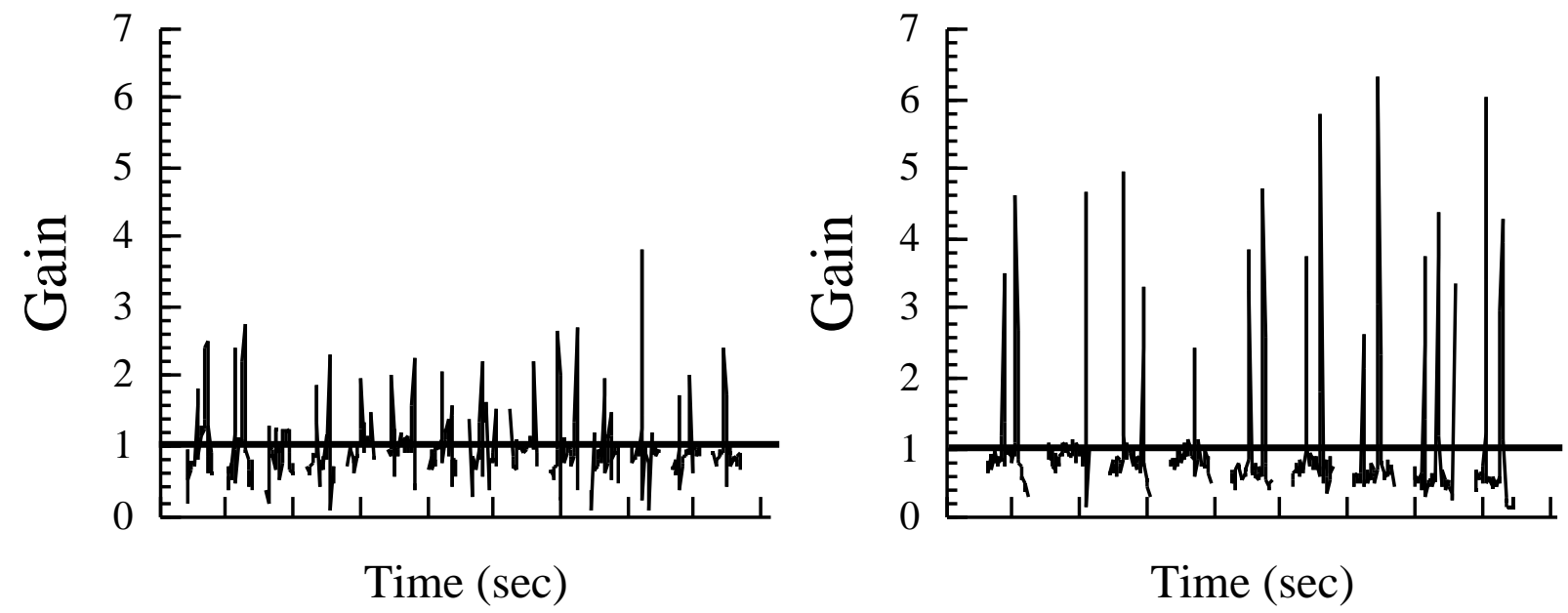

Figure 3. Shown are the gain values (eye/target velocity) for the recordings shown in Figure 2. Sober observers consistently show gains around 1.0 (0.97 in left panel) while intoxicated observer have gain values much less than 1.0 (0.68 in right panel). The spikes denoting high eye velocities are fast saccadic eye movements.

Alcohol intoxication raised observer thresholds in both the active and passive motion parallax conditions (Figure 4). Even observers having peak BAC well below $0.1 \%$ and unaffected thresholds reported subjective difficulty with the motion parallax task. In contrast, no threshold elevation was seen in the binocular stereopsis test, although the test had a floor of 20 arc sec.

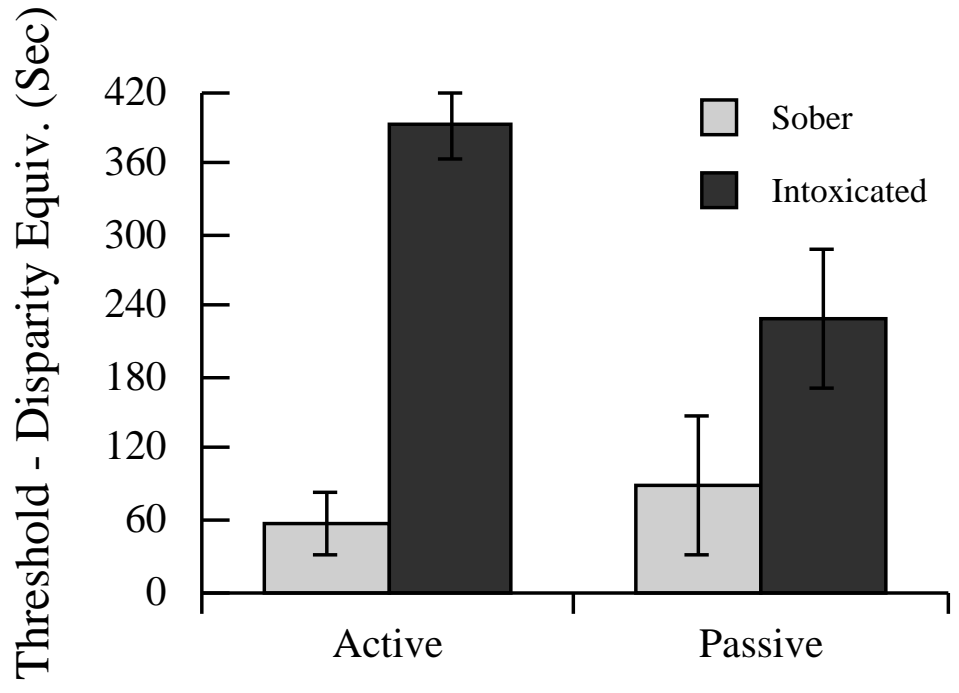

\section{Motion Parallax}

Figure 4. Both active and passive motion parallax had low thresholds in the sober condition and greatly increased thresholds in the intoxicated condition. 


\section{DISCUSSION}

The current study shows that an important perceptual system for driving is compromised by alcohol intoxication. This effect is due to the already well known effects of alcohol on slow eye movements. Although visual depth perception relies on many different types of information, motion parallax is undoubtedly one of the most important during rapid translation through the environment such as driving. Perhaps a quick decision based on faulty or inadequate perceptual information is a critical component in the driving accident risk with alcohol intoxication.

\section{ACKNOWLEDGEMENTS}

Research supported by NIH/NEI R01 EY12541. Thanks to Benita Nordenstrom and Michael Himle for help in data collection and analysis.

\section{References}

Belton, H. (1987). Lateral nystagmus: A specific diagnostic sign of ethyl alcohol intoxication. N.Z. Med. J., 100, 534-535.

Forkiotis, C. J. (1986). Horizontal gaze nystagmus as part of roadisde testing. Am. J. Optom. Physiol. Opt., 63, 1000.

Good, G. W. \& Augsburger, A. R. (1986). Use of horizontal gaze nystagmus as part of roadside sobriety testing. Am. J. Optom. Physiol. Opt., 63, 467-471.

Holdstock L. \& de Wit, H. (1999). Ethanol impairs saccadic and smooth pursuit eye movements without producing self-reports of sedation. Alcohol. Clin. Exp. Res,. 23, 664-672.

Nawrot, M. (1997). Role of slow eye movements in depth from motion parallax. Invest. Ophthal.Vis. Sci., 38, 3230.

Nawrot, M. (2000). Veiwing distance, eye movements, and the perception of relative depth from motion parallax. Invest. Ophth. Vis. Sci., 41233.

Nicholson, M. E., Andre, J. T., Tyrrell, R. A., Wang, M. \& Leibowitz, H. W. (1995). Effects of moderate dose alcohol on visual contrast sensitivity for staionary and moving Targets. J. Stud. Alcohol, 56, 261-266.

Pearson, P., \& Timney, B. (1998). Effects of moderate blood alcohol concentrations on spatial and temporal contrast sensitivity. J. Alcohol Studies, 59, 163-173.

Rogers, B. J. \& Graham, M. E. (1979). Motion parallax as an independent cue for depth perception. Perception, $\underline{8}, 125-134$.

Stapleton, J. M., Guthrie, S. \& Linnoila, M. (1986). Effects of alcohol and other psychotropic drugs on eye movementsL relevance to traffic safety. J. Stu. Alcohol, 47, 426-432,

Tiffany, D. V. (1986). Optometric expert testimony: Foundation for the horizontal gaze nystagmus test. J. Am. Optom. Assoc., 57, 705-708.

Wallgren, H. \& Barry, H. (1970). Action and alcohol. Elsevier Biomedical Press: Amsterdam.

Watten, R. G. \& Lie, I. (1996). Visual functions and acute ingestion of alcohol. Opthal. Physiol. Opt., 16, 460-466,

Wegner, A. J. \& Fahle, M. (1999). Alcohol and visual performance. Prog. NeuroPsychopharmacology \& Biol. Psychiat, 23, 465-482. 